\title{
AIP
}

\section{Impedance e-tongue instrument for rapid liquid assessment}

Flávio P. A. Cabral, Bruno B. Bergamo, Cleber A. R. Dantas, A. Riul, and José A. Giacometti

Citation: Rev. Sci. Instrum. 80, 026107 (2009); doi: 10.1063/1.3084210

View online: http://dx.doi.org/10.1063/1.3084210

View Table of Contents: http://rsi.aip.org/resource/1/RSINAK/v80/i2

Published by the AIP Publishing LLC.

\section{Additional information on Rev. Sci. Instrum.}

Journal Homepage: http://rsi.aip.org

Journal Information: http://rsi.aip.org/about/about_the_journal

Top downloads: http://rsi.aip.org/features/most_downloaded

Information for Authors: http://rsi.aip.org/authors

\section{ADVERTISEMENT}

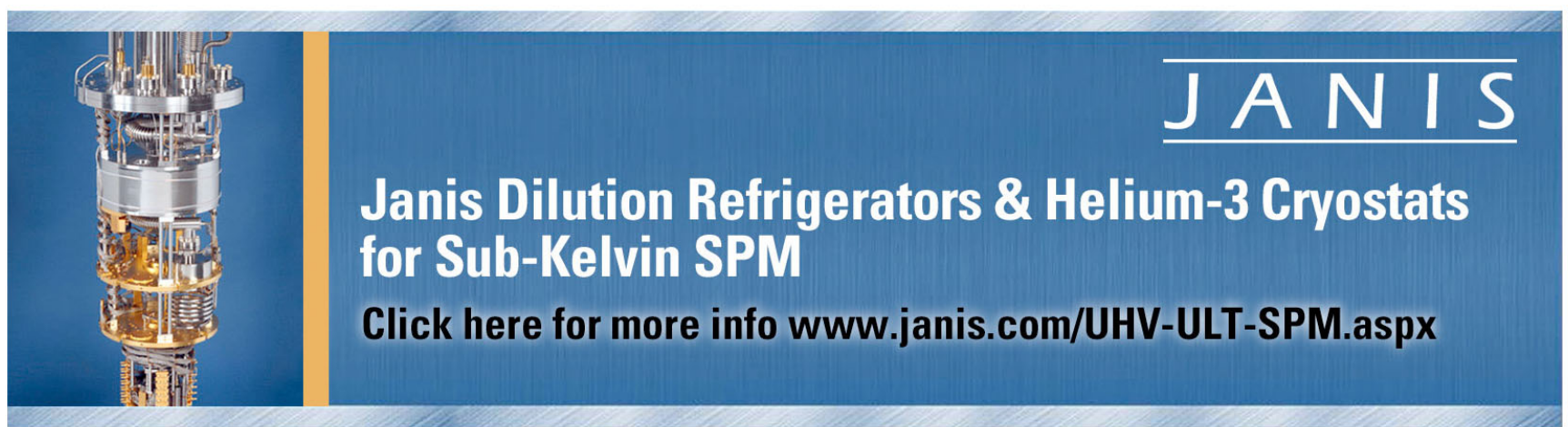




\title{
Impedance e-tongue instrument for rapid liquid assessment
}

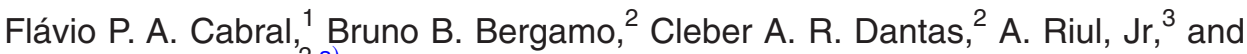 \\ José A. Giacometti ${ }^{2, a)}$ \\ ${ }_{1}^{1}$ FIPP, Universidade do Oeste Paulista, 19050-920, Presidente Prudente, Brazil \\ ${ }^{2}$ Faculdade de Ciências e Tecnologia, UNESP, 19060-900, Presidente Prudente, Brazil \\ ${ }^{3}$ Universidade Federal de São Carlos, Campus Sorocaba, CP 3031, 18052-780, Brazil
}

(Received 6 September 2008; accepted 28 January 2009; published online 24 February 2009)

\begin{abstract}
We present a compact and easy to handle instrument developed to perform rapid analysis of liquids utilizing an "electronic tongue" system. Briefly, the e-tongue used here is based on impedance measurements of an array of sensing units fabricated with ultrathin films of different materials deposited onto gold interdigitated electrodes. The instrument has the capability of measuring up to eight different sensor sets, each comprising an array of eight sensing units, and can perform a series of measurements in less than half of an hour. Additionally, there is a user-friendly software interface for instrument control, allowing the statistical correlation of samples using principal component analysis. () 2009 American Institute of Physics. [DOI: 10.1063/1.3084210]
\end{abstract}

The artificial tongue concept is based on the application of an array of nonspecific sensors with wide sensitivity toward several media components (broad cross sensitivity), largely employed in the analysis of tastants, ${ }^{1}$ medicines, ${ }^{2,3}$ foodstuffs, ${ }^{4-6}$ and pollutants. ${ }^{7,8}$ The impedance-sensing units made from ultrathin films physically interact with analytes in solution according to their electrical nature, providing a fingerprint of the solution similar to the global selectivity concept in the human tongue.

We present here a low-cost, compact, and easy to handle e-tongue instrument developed to perform reliable measurements in liquid samples. The instrument is able to perform reliable measurements in up to eight distinct electronic tongues immersed in quite different samples (mineral waters, coffee, tastants, beverages, etc.) ${ }^{10}$ with precision comparable to commercial impedance analyzers.

Figure 1 is a simplified schematic of the hardware used, mainly composed of a signal generator, amplifiers, multiplexers, digital acquisition (DAQ) board from National Instruments Corp., analogic/digital converter, and a notebook computer (not shown). The DAQ-NI6036E (Ref. 11) board has an A/D converter with resolution of 16 bits, acquisition rate of 200 ksamples per second, and digital outputs. It is a Personal Computer Memory Card Internation Association (PCMCIA) card acquisition board for a notebook computer, therefore, suitable for on-site measurements. The hardware comprises

a signal generator (XR8038, Exar Corporation) to apply a $1 \mathrm{kHz}, 150 \mathrm{mV}$ sinusoidal voltage $\left(V_{\text {in }}\right)$ to the sensing units, a current amplifier (TL074, STMicroelectronics) for conditioning the electric current signal generated through the sensing unit $\left(V_{\text {out }}\right)$, voltage amplifiers (TL074) to match the amplitudes of the signals output of the current amplifier and of the generator to the input of DAQ, two analog multiplexers (MAX313 from Maxim-Dallas Semiconductor) and a logic circuit to turn on/off multiplexer switches (not shown in

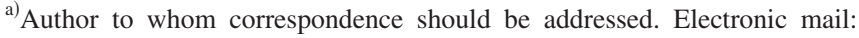
Giacometti@fct.unesp.br.
}

Fig. 1). The first multiplexer is used to address each e-tongue and the second to address each sensing unit. The logic circuit was connected to the digital output of the NIDAQ enabling the software to determine which sensing unit should be measured. The MAX313 multiplexer has a low leakage current that allows the measurement of capacitances over the range from 1 to $100 \mathrm{nF}$, usually found in sensing units in e-tongues. ${ }^{1}$

The main capability of the instrument is based on its software that was developed using the integrated development environment Borland $\mathrm{C}++$ Builder. For example, after connecting different e-tongues to the instrument, the software checks if the sensing units used in each e-tongue are properly connected and accurately working, adding a specific identification to each of them. Measurement conditions can also be defined by the user such as holding time before the start of data acquisition, number of measurements to be performed in each e-tongue, and also the number of e-tongue sets to be employed. In the next step the software starts measurements, scanning all sensing units connected, and digitizing the alternating electric current signal and applied voltage to each sensing unit. Once measurements are finished the user can either save the data in EXCEL format or proceed to principal component analysis (PCA) analysis, with the PCA plots displayed on the computer screen. The software also has the capabilities of zooming on a specific area of the PCA plot, printing, and exporting the graphic.

Current and voltage ac signals were digitized taking 50 points in each period of the signals. In order to improve the signal to noise ratio ten cycles of each signal were acquired and the mean value of each digitized point was calculated. The process is repeated 15 times with mean values recalculated. The acquisition time for each sensing unit at $1 \mathrm{kHz}$ takes $\sim 0.2 \mathrm{~s}$. Determination of the sensor impedance was made by applying discrete Fourier transform to the digitized signals. From amplitude and phase difference between current and voltage, the complex impedance of each sensing unit was obtained and its electric capacitance was calculated. The current amplifier gain should be considered in capaci- 


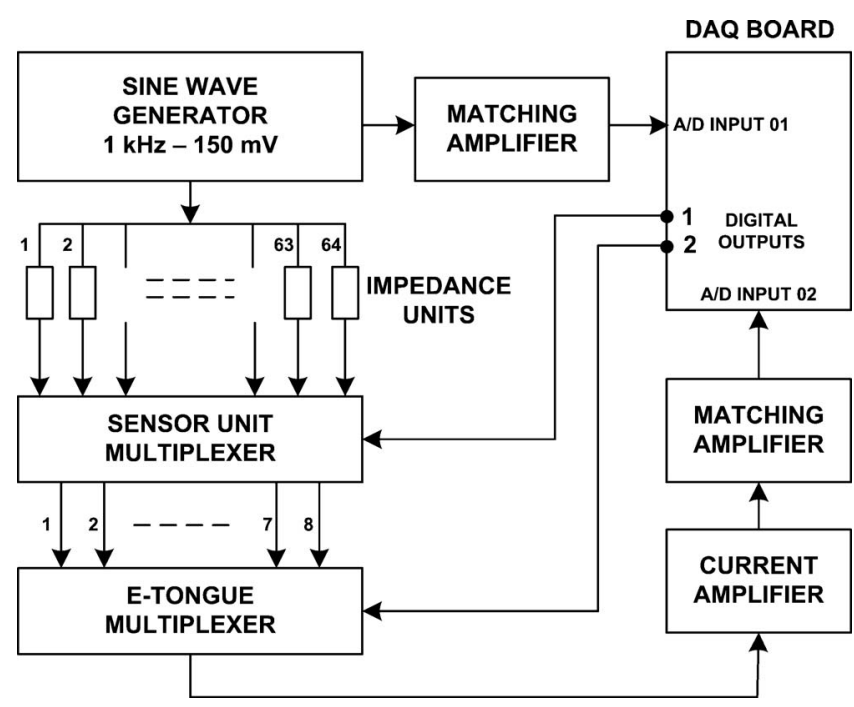

FIG. 1. Simplified diagram of the setup hardware.

tance determination in order to get absolute values, which can be introduced or modified through the software by the user. This possibility allows the calibration of the instrument by the user whenever necessary using a reference capacitor or a resistor.

The instrument has the capability to measure capacitances over the range of $2-120 \mathrm{nF}$, with high precision (error lower than 1\%), corresponding to an electric impedance range of 1.5-70 $\mathrm{M} \Omega$, suitable for sensors usually employed in e-tongues using thin films. It can scan 64 sensing units, presenting the results in a very short period of time (of the order of a few minutes). Moreover, if the capacitance cannot be measured with a precision less than $1 \%$, the software identifies the sensing unit as deactivated.

In order to evaluate the e-tongue instrument performance, five-layer Langmuir-Blodgett films of polypyrrole, a poly(styrene-co-methyl methacrylate) ionomer, lignin, and a perylene derivative, and a three-bilayer chitosan/poly(4styrenesulfonate) self-assembled film were deposited onto gold interdigitated electrodes $(10 \mu \mathrm{m}$ width, $10 \mu \mathrm{m}$ apart, displaying 50 pairs of digits) and used as individual sensing unit of an e-tongue array.

The good agreement between the experimental points in the PCA plot in Fig. 2 is indicative of the precision achieved by the system when compared with the Solartron analyzer, despite the error difference previously mentioned. Another advantage of our e-tongue instrument is the integrated automatic generation of PCA plots of the samples analyzed without external software. Moreover, multiplexation allows a rapid assessment of the data permitting a task that took previously a couple of hours to complete with one e-tongue array to be now executed in less than $40 \mathrm{~min}$. The main difference among the water samples tested was the ion concentration, and as observed in Fig. 2, it can be correlated through the first principal component evidenced by a marked displacement to the right hand side in the PCA plot with increasing ion concentration, i.e., from Milli-Q to tap water. The system responds quite well also to more complex liquid samples as exemplified in Fig. 3, in which a blind test was performed in Latex samples used in the production of chew gums kindly provided by Petroflex (Petrópolis, RJ-Brazil).

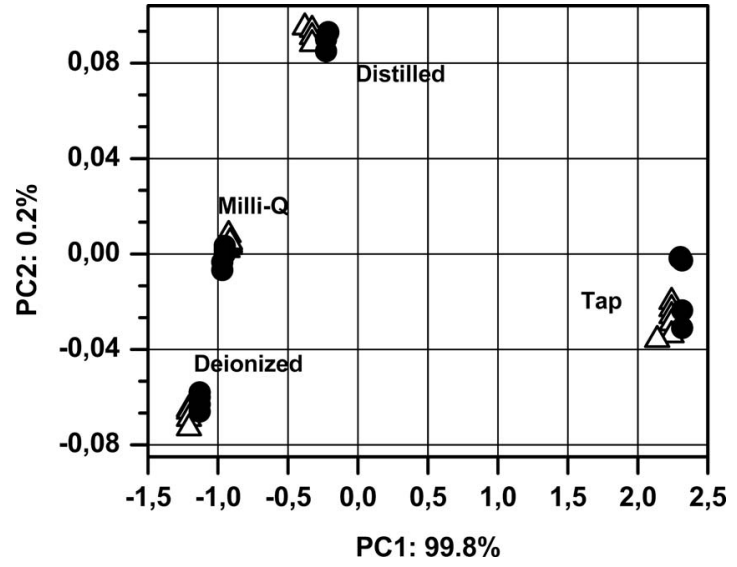

FIG. 2. PCA plot of aqueous solutions acquired with the e-tongue instrument $(\triangle)$ and the Solartron 1260A $(\odot)$. Results are the mean values of five measurements.

The samples analyzed were obtained from the washing process of chew gums during their fabrication. The main difference was the bitter taste, which was stronger is some of them.

The main requirement of an e-tongue for commercial use is that replacement of sensing elements with the same characteristics, that is, several sensing units prepared with the same ultrathin film should have the same capacitive response. Therefore, capacitance variations are due to the difficulty of producing interdigitated electrodes having exactly the same capacitance and also to the reproducibility of the ultrathin film deposition process. Since our e-tongue instrument performs measurements in up to 64 sensing units, it can be readily employed to select and classify groups of similar sensing units having the same capacitance values for a given measuring condition.

In Fig. 4 we show capacitance values acquired in four distinct e-tongue arrays immersed in Milli-Q water. Each array contains five sensing units fabricated from ultrathin films as described in the text. Since the sensing units were prepared with the same materials of similar thickness, they should have comparable capacitance values, which in practice is not so easy to achieve as shown in Fig. 4. The observed differences in the measured capacitance values of the

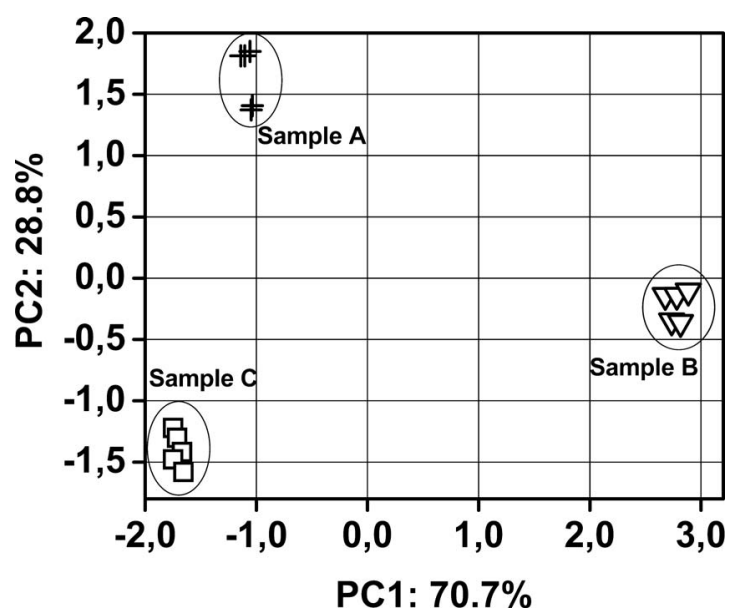

FIG. 3. PCA plot distinguishing water samples from the washing process of chew gums. 


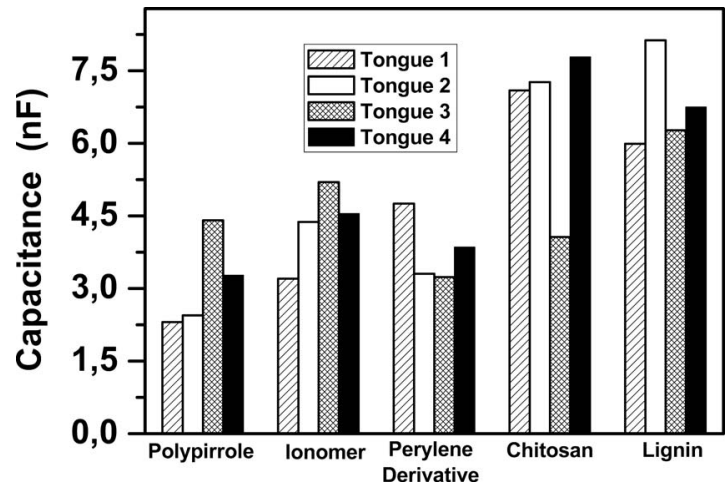

FIG. 4. Capacitance values of the individual sensing units measured in four distinct e-tongues. The polymers used for the fabrication of the units are given in the figure.

sensing units are attributed to variations in capacitances of the bare interdigitated electrodes and by the fact that ultrathin films cannot be obtained with exactly the same characteristics. The idea here was to demonstrate the difficulties involved in producing similar sensing units based on nanostructured thin films, which might hamper potential applications. Therefore, the e-tongue instrument is helpful in verifying rapidly if the readability of the interdigitated electrodes and quality of film fabrication are maintained in distinct setups.

A compact and easy to handle instrument designed for signal generation, multiplexation, and data acquisition in an "electronic tongue" based on impedance measurements of ultrathin films of different materials deposited on gold interdigitated electrodes was developed to perform rapid analysis in liquid samples. The system has a precision comparable to a commercial impedance analyzer, but achieved at a much lower cost. Additionally, the instrument has high flexibility and the capability of measuring up to eight different electronic tongues, each comprising eight impedance units. The system allows a series of measurements to be performed in a short period of time (few minutes) and we believe that it will allow easy integration into commercial sensor systems in the near future.

The authors are grateful to FAPESP, IMMP, and CNPq for the financial support, and also to LNLS for the interdigitated electrodes used (Project No. MIC 5508).

${ }^{1}$ A. Riul, Jr., R. R. Malmegrim, F. J. Fonseca, and L. H. C. Mattoso, Biosens. Bioelectron. 18, 1365 (2003).

${ }^{2}$ E. Tsuji, T. Uchida, A. Fukui, R. Fujii, and H. Sunada, Chem. Pharm. Bull. (Tokyo) 54, 310 (2006).

${ }^{3}$ A. Gutés, F. Céspedes, R. Cartas, S. Alegret, M. del Valle, J. M. Gutierrez, and R. Muñoz, Chemom. Intell. Lab. Syst. 83, 169 (2006).

${ }^{4}$ Y. G. Vlasov, A. V. Legin, and A. M. Rudnitskaya, Usp. Khim. 75, 141 (2006).

${ }^{5}$ M. Scampicchio, S. Benedetti, B. Brunetti, and S. Mannino, Electroanalysis 18, 1643 (2006)

${ }^{6}$ V. Parra, A. A. Arrieta, J. A. Fernández-Escudero, M. Iñiguez, J. A. de Saja, and M. L. Rodríguez-Méndez, Anal. Chim. Acta 563, 229 (2006).

${ }^{7}$ A. Gutes, F. Cespedes, S. Alegrete, and M. del Valle, Biosens. Bioelectron. 20, 1668 (2005).

${ }^{8}$ Y. Vlasov, A. Legin, and A. Rudnitskaya, Sens. Actuators B 44, 532 (1997).

${ }^{9}$ K. Toko, Meas. Sci. Technol. 9, 1919 (1998).

${ }^{10}$ J. A. Giacometti, F. P. Albuquerque, A. Riul, Jr., and B. B. Bergamo, Brazilian Patent No. PI 0700292-0, INPI (pending).

${ }^{11}$ National Instruments Corporation, Austin, Texas USA (www.ni.com). 